\title{
NUEVOS DATOS SOBRE EL REGISTRO MALACOLÓGICO DEL PLEISTOCENO SUPERIOR-HOLOCENO DE LA CUEVA DE NERJA (MÁLAGA, ESPAÑA)
}

Trabajo presentado a las XVIII Jornadas de Paleontología y

II Congreso Ibérico de Paleontología

Universidad de Salamanca

Salamanca, 24-29 de Septiembre de 2002

\author{
M. Carmen LOZANO-FRANCISCO ${ }^{1}$, José Luis \\ VERA-PELÁEZ ${ }^{1}$, María Dolores SIMÓN \\ VALLEJO Y Miguel CORTÉS SÁNCHEZ ${ }^{3}$
}

\footnotetext{
${ }^{1}$ Museo Municipal de Paleontología de Estepona. Avda. Matías Prats s/n. Plaza de Toros.29680 Estepona (Málaga). e-mail: delcultu@teleline.es

${ }^{2}$ Fundación Cueva de Nerja. C/ Carretera de Maro, s/n. 29787 Nerja (Málaga).e-mail: msimon@cuevanerja.es

3 Área de Prehistoria. Universidad de Córdoba. Plaza Cardenal Salazar, s/n, 14071-Córdoba. e-mail: mm.cosi@teleline.es
}

Lozano-Francisco, M. C., Vera-Peláez, J. L., Simón Vallejo, M. D. y Cortés Sánchez, M. 2004. Nuevos datos sobre el registro malacológico del Pleistoceno Superior-Holoceno de la Cueva de Nerja (Málaga, España). [Contribution to the knowledge of the mollusks record of Upper Pleistocene-Holocene in Nerja's cave (Málaga, Spain).] Revista Española de Paleontología, 19 (2), 215-228. ISSN 0213-6937.

\begin{abstract}
A systematic, autoecologic and anthropic study of the mollusks and invertebrates of arqueologic intervention of Sala del Vestibulo of Cueva de Nerja (Málaga) has been accomplished. The sediments have been included between Upper Pleistocene and Holocene (21,000-6,000 B.P.). 48 species of invertebrates, 45 of them are mollusks: two species of scaphopods, 15 of bivalves and 30 of gastropods have been identified. The majority of gastropods are marines, of intermareal-rocks and one species from rivers (Melanopsis laevigata Lamarck, 1818) and other five species of Helicidae (Pulmonata) are continentals around of the cave. The rest of invertebrates are a echinoids (Echinodermata): Paracentrotus lividus (Linné, 1758) and two Crustacea (Balanus sp. and Brachiura sp.). The environment of the coast was mainly a beach in Upper Paleolitic (Würm III-IV to Tardiglacial), so the hunting of infralitoral mollusks was of sand-mud. Three species of mollusks were utilized exclusively as ornamental elements: two scaphopods [Antalis inaequicostatum (Dautzenberg, 1896) and Antalis vulgare (Linné, 1758)] and a deep red gastropod [Jujubinus exasperatus (Pennant, 1777)].
\end{abstract}

Keywords: Mollusca, Upper Pleistocene, Holocene, Nerja's cave, Málaga.

\section{RESUMEN}

Se ha realizado un estudio sistemático, autoecológico y antrópico de los moluscos e invertebrados recuperados en una intervención arqueológica en la Sala del Vestíbulo de la Cueva de Nerja (Málaga). Los sedimentos en los que se han recuperado se encuadran entre el Pleistoceno superior-Holoceno (21.000-6.000 B.P.). Hemos identificado 48 especies de invertebrados, de las que 45 son moluscos: dos especies de escafópodos, 15 de bivalvos y 30 de gasterópodos. Estos últimos son en su mayoría marinos, de la zona intertidal rocosa, si bien hay una especie de ambiente fluvial (Melanopsis laevigata Lamarck, 1818) y otras cinco de helícidos (pulmonados), de procedencia continental, recogidos en los alrededores de la cueva. Los otros invertebrados son un erizo de mar (Echinodermata): Paracentrotus lividus (Linné, 1758) y dos crustáceos (Balanus sp. y Brachiura sp.). En el Paleolítico Superior (Würm III-IV a Tardiglacial) el ambiente costero sería fundamentalmente de playa, lo que propiciaría la captura de fauna infralitoral arenosa-fangosa. Al menos tres especies de moluscos fueron utilizados en exclusiva como elementos ornamentales, los dos escafópodos: [Antalis inaequicostatum (Dautzenberg, 1896) y Antalis vulgare (Linné, 1758)] y un gasterópodo, Jujubinus exasperatus (Pennant, 1777), de fuerte color rojo.

Palabras clave: Mollusca, Pleistoceno Superior, Holoceno, Cueva de Nerja, Málaga. 


\section{INTRODUCCIÓN}

La Cueva de Nerja (Fig. 1) constituye uno de los yacimientos más interesantes para el estudio del aprovechamiento por parte de las comunidades humanas de los recursos marinos durante el Pleistoceno Superior y Holoceno antiguo (ver Sanchidrián et al., 1996; Serrano et al., 1997, 1998; Aura et al., 2002 a, b).

Así, tanto a nivel cualitativo como cuantitativo, el repertorio malacológico recuperado en distintas campañas de excavación en este yacimiento, lo erigen en el más importante conocido hasta la fecha para este tipo de documentación dentro del contexto mediterráneo peninsular.

Sin embargo, algunas de las intervenciones arqueológicas llevadas a cabo sobre este enclave permanecían inéditas por diversas circunstancias. En este sentido, el presente trabajo aborda el estudio de la malacofauna recuperada en la Cueva de Nerja durante las campañas de exca- vación que llevó a cabo A. M. de la Quadra Salcedo, que permanecían sin publicar.

Los trabajos de esta investigadora se desarrollaron en dos campañas de excavación (1962 y 1963), afectando al relleno sedimentario de la Sala del Vestíbulo de la Cueva de Nerja, donde diseñó un corte con una superficie de excavación de $14 \mathrm{~m}^{2}$, que seguía a su vez un corte iniciado con anterioridad (1960) por el prof. Manuel Pellicer (ver Pellicer Catalán, 1997), el cual sólo trabajó sobre los niveles más superficiales (Calcolítico y Neolítico).

Diversas vicisitudes personales hicieron que A. M. de la Quadra abandonara la investigación y que los materiales se dispersaran en diversas colecciones e instituciones, de modo que al correr el tiempo se perdió incluso constancia de su localización concreta (Cortés et al., 2002).

En este contexto, desde la vocalía de Arqueología de la Comisión Científica Asesora del Patronato de la Cueva de Nerja, se inició a mediados de los años '90 un proyec-
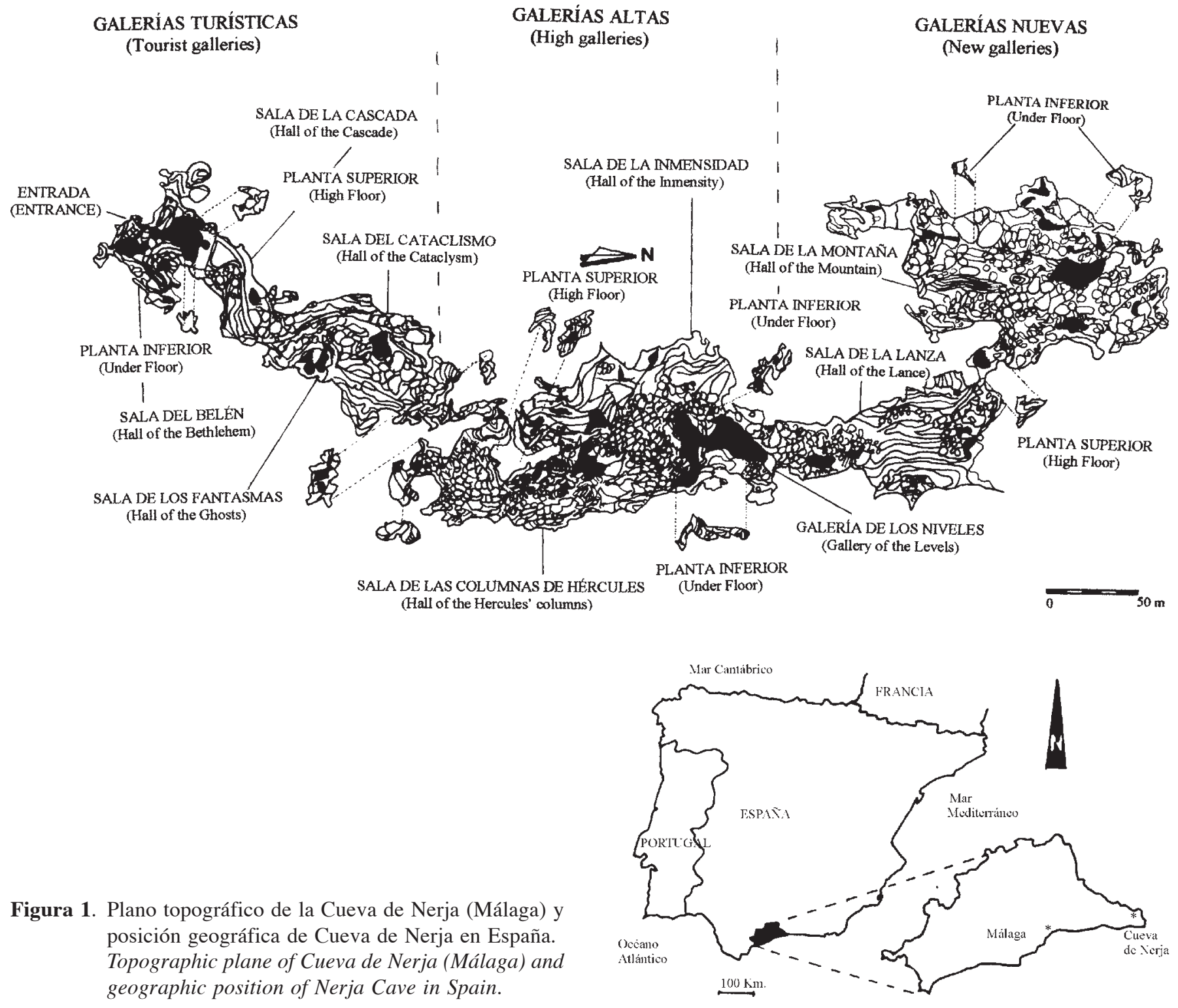

GALERÍAS NUEVAS (New galleries) 
to de localización de los materiales de las antiguas excavaciones llevadas a cabo en la cavidad, auspiciando para ello el desarrollo de un proyecto de investigación coordinado por el prof. José Luis Sanchidrián y dirigido por uno de nosotros (Miguel Cortés Sánchez).

Así, en 1996, localizamos en el Museo Provincial de Málaga diversas cajas con materiales arqueológicos procedentes de la intervención de esta investigadora y, a través de un colaborador en la intervención de campo (D. José Luis Barbero de Miguel), pudimos acceder a parte de la documentación de excavación (planimetrías, fotografías, anotaciones al diario de campo, etc).

En este sentido, el material analizado aquí se encontraba todavía envuelto en los contenedores empleados durante la intervención arqueológica, que seguía una agrupación por cuadrícula y nivel identificado.

Durante los años '80 tuvieron lugar nuevas excavaciones en la Sala del Vestíbulo de la Cueva de Nerja, dirigidas esta vez por el prof. F. Jordá, sobre áreas no trabajadas en las campañas de 1962-1963. A tenor de la documentación recuperada pudo finalmente articularse la secuencia cronológica y cultural de esta sala (Aura et al., 1998).

Dada la coincidencia entre las secuencias documentadas en ambos conjuntos de intervenciones (Cortés et al., 2002), hemos mantenido la segmentación propuesta por Aura et al. (1998) a la hora de analizar los materiales malacológicos de las excavaciones de A. M. de la Quadra (Tabla 1).

Los resultados del estudio de los nuevos materiales que presentamos aquí vienen a unirse a otros obtenidos en trabajos de investigación sobre los moluscos y fauna de invertebrados de la Cueva de Nerja procedentes de cuatro cortes estratigráficos realizados en la Cueva de Nerja en las salas de la Mina y de la Torca y otro realizado en la propia sala del Vestíbulo (Jordá Pardo, 1981, 1982, 1983, 1984-1985, 1986; Lozano-Francisco y Vera-Peláez, 1995; Serrano et al., 1995, 1996, 1997, 1998; Aura et al., 2002b; Lozano-Francisco et al., 2003; Vera-Peláez et al., 2003)

\begin{tabular}{|c|c|c|c|c|c|}
\hline $\begin{array}{c}\text { Estratos } \\
\text { A. M. Quadra }\end{array}$ & Unidades & $\begin{array}{c}\text { Edad en miles } \\
\text { de años B.P. }\end{array}$ & \multirow{2}{*}{ Cuaternario } & \multirow{2}{*}{$\begin{array}{c}\text { Cultura } \\
\text { material }\end{array}$} \\
\hline Cortés et al.,2002 & \multicolumn{2}{|c|}{ Aura et al., 1998 } & & Neolítico \\
\hline I-II-III & 5 & $7,5-6$ & \multirow{2}{*}{ Holoceno } & Epipaleolitico \\
\cline { 3 - 3 } IV & 4 & $7-11$ & & Tardiglacial & Magdaleniense \\
\hline V-VI-VII & 3 & $11,5-12,5$ & Würm III-IV & Solutrense \\
\hline VIII-IX-X & 2 & $17-20$ & Wün III & Gravetiense \\
\hline XI-XII-XII & 1 & $21-25$ & Würm III \\
\hline
\end{tabular}

Tabla 1. Correlación de la secuencia estratigráfica de las excavaciones de A. M. de la Quadra y unidades cronoculturales de la Sala del Vestíbulo propuesta por Aura et al. (1998).

Correlation between the stratigraphic sequence of $A$. M. de la Quadra Salcedo and the Chronocultural sequence of Sala del Vestíbulo from Aura et al. (1998). un avance de este trabajo ha sido presentado en el II Congreso Ibérico de Paleontología (Lozano-Francisco et al., 2002).

En lo relativo a los materiales procedentes de las excavaciones de 1962-1963, los restos de malacofauna se distribuyen entre los niveles NV-XI y NV-I, es decir, por su correlación con la secuencia conocida en la Sala del Vestíbulo entre $c a .21 .000$ B.P. y $c a$. 6.000 B.P. (Aura et al., 1998) (vid. Tabla 1).

La acumulación de fauna de invertebrados en estos niveles no sólo se debe atribuir a la utilidad alimentaria de los mismos, sino que aparece claramente ligada en unos casos a una función ornamental, en otros no se debe de descartar su utilización como ajuar funerario y en ocasiones a incorporaciones a los depósitos sin concurrir el factor humano.

Así, la mayor parte de los ejemplares analizados tendrían un uso alimentario, mientras que a nivel interno en los diferentes segmentos crono-culturales existen variaciones en la aportación de las diferentes especies a la dieta, aunque en un contexto general en el que la aportación más cuantiosa corresponde a mejillones [Mytilus edulis (Linné, 1758)] y patéllidos.

No obstante, existen una serie de moluscos que debieron tener una función ornamental, pues se trata de conchas muy vistosas, no comestibles y en su mayoría de pequeña talla, como es el caso de los escafópodos Antalis inaequicostatum (Dautzenberg, 1891), Antalis vulgare (Da Costa, 1776) o del gasterópodo Jujubinus exasperatus (Pennant, 1777) de un fuerte color rojo. Además, sobre buena parte de este tipo de ejemplares aparecen realizados diferentes tipos de perforaciones relacionadas con su transformación en colgantes. En lo relativo a la utilización de carácter simbólico de estos recursos, existe ya un tratamiento en algunos trabajos sobre la Cueva de Nerja (p. ej. Jordá, 1982; Cotino Villa y Soler Mayor, 1998), aspecto que no obstante se verá enriquecido cualitativa y cuantitativamente con las nuevas colecciones identificadas, cuyos ítems están siendo evaluados tanto desde un punto de vista tecnológico (formas de fabricación, manipulación de las conchas, etc) como de utilización diferencial a nivel diacrónico (modificaciones porcentuales de las especies transformadas en adornos).

Por último, los datos procedentes del nuevo registro malacológico que expondremos a continuación añaden información a las deducciones sobre la dinámica glacioeustática de la línea de costa en el sector donde se ubica la Cueva de Nerja; asimismo, nos introducimos en los patrones de adquisición de estos recursos por parte de la comunidades prehistóricas que los aprovecharon, a partir de la identificación del tipo de hábitat donde se recogieron, en función de que la malacofauna fuera de origen continental, fluvial o marino y, en este caso, si procedía de ambientes rocosos, fangosos o arenosos o si pertenecía a la zona mesolitoral o la infralitoral, e incluso la batimetría de estas especies. 


\begin{tabular}{|c|c|c|c|}
\hline FILUM & Clase & Orden & Especie \\
\hline \multirow{2}{*}{ Arthropoda } & Malacostraca & Decapoda & Brachiura sp. \\
\hline & Cirripedia & Thoracica & Balanus sp. \\
\hline Echinodermata & Echinoidea & Echinoidea & Paracentrotus lividus (Linné, 1758) \\
\hline \multirow{11}{*}{ Mollusca } & Scaphopoda & Dentalioida & \begin{tabular}{|l|} 
Antalis inaequicostatum (Dautzenberg, 1891) \\
Antalis vulgare (Da Costa, 1776) \\
\end{tabular} \\
\hline & & Arcoida & Glycymeris (Glycymeris) sp. \\
\hline & & Mytiloida & \begin{tabular}{|l|} 
Mytilus (Mytilus) edulis Linné, 1758 \\
Modiolus (Modiolus) modiolus (Linné, 1758) \\
\end{tabular} \\
\hline & & Ostreoida & $\begin{array}{l}\text { Ostrea (Ostrea) edulis Linné, } 1758 \\
\text { Pecten (Pecten) maximus (Linné, 1758) } \\
\text { Pecten (Pecten) jacobaeus (Linné, 1758) } \\
\text { Anomia ephippium Linné, } 1758\end{array}$ \\
\hline & Bivalvia & Veneroida & $\begin{array}{l}\text { Rudicardium tuberculatum (Linné, 1758) } \\
\text { Cerastoderma edule (Linné, 1758) } \\
\text { Mactra stultorum (Linné, 1758) } \\
\text { Ensis ensis } \text { (Linné, 1758) } \\
\text { Venus verrucosa Linné, 1758 } \\
\text { Callista chione (Linné, 1758) } \\
\text { Irus irus (Linné, 1758) } \\
\text { Tapes decussatus (Linné, 1758) }\end{array}$ \\
\hline & \multirow{6}{*}{ Gastropoda } & Docoglossa & $\begin{array}{l}\text { Patella caerulea Linné, } 1758 \\
\text { Patella ferruginea Gmelin, } 1791 \\
\text { Patella intermedia Knapp in Murray, } 1857 \\
\text { Patella nigra Da Costa, } 1771 \\
\text { Patella rustica Linné, } 1758 \\
\text { Patella ulyssiponensis Gmelin, } 1791\end{array}$ \\
\hline & & Vetigastropoda & $\begin{array}{l}\text { Gibbula richardi (Payraudeau, 1826) } \\
\text { Gibbula sp. } \\
\text { Monodonta turbinata (von Born, 1778) } \\
\text { Jujubinus exasperatus (Pennant, 1777) }\end{array}$ \\
\hline & & Neotaenioglossa & $\begin{array}{l}\text { Littorina sp. } \\
\text { Melanopsis laevigata (Lamarck, 1818) } \\
\text { Turritella monterosatoi Kobelt, 1888 } \\
\text { Charonia lampas lampas (Linné, 1758) } \\
\text { Cymatium corrugatum (Lamarck, 1816) } \\
\text { Cabestana cutacea (Linné, 1758) }\end{array}$ \\
\hline & & Neogastropoda & $\begin{array}{l}\text { Nucella lapillus (Linné, 1758) } \\
\text { Stramonita haemastoma (Linné, 1758) } \\
\text { Columbella rustica (Linné, 1758) } \\
\text { Cyclope donovania Risso, 1826 } \\
\text { Nassarius corniculus (Olivi, 1792) } \\
\text { Conus mediterraneus } \text { Hwass in Bruguière, } 1791\end{array}$ \\
\hline & & Divasibranchia & Siphonaria pectinata (Linné, 1758) \\
\hline & & Stylommatophora & $\begin{array}{l}\text { Rumina decollata (Linné, 1758) } \\
\text { Sphincterochila hispanica (Westerlund, 1886) } \\
\text { Iberus alonensis (Férussac, 1821)* } \\
\text { Iberus marmoratus (Férussac, 1821)* } \\
\text { Otala (Otala) lactea (Müller, 1774) } \\
\end{array}$ \\
\hline
\end{tabular}




\section{MATERIAL Y MÉTODOS}

Como hemos expuesto, la colección sobre la que hemos trabajado procede de la excavación de $14 \mathrm{~m}^{2}$, con una potencia sedimentaria que superaba los $2 \mathrm{~m}$, llevada a cabo en la sala del Vestíbulo de la Cueva de Nerja entre los años 1962 y 1963.

Los materiales malacológicos, que había sido separados del resto de objetos en la propia excavación, permanecían en los contenedores habilitados en su momento, sin limpiar ni tratar, aunque conservando de forma clara su cuadrícula y estrato de procedencia. Así pues, una vez desembalado el material fue lavado, clasificado e identificado, contabilizado el número de especies y su abundancia por niveles.

En el caso de los bivalvos, por presentar dos valvas, la estimación del número mínimo de individuos (NMI) es el resultado de sumar todas las valvas/2, en los ejemplares fragmentados sólo se contaron umbos para evitar contar un mismo ejemplar varias veces, mientras que para los gasterópodos fragmentados se procedió del siguiente modo: de los patéllidos, se contaron los ápices y en el resto las columelas. Así pues, la colección analizada consta de un NMI de 5.348.

Una vez realizada la sistemática, identificamos el tipo de hábitat que representaban, los tipos de sustrato y de alimentación (autoecología), reconstrucción del medio, acción antrópica sobre los moluscos (relacionados con modificaciones para su uso como ornamento, alimento, etc).

Los resultados se han presentado en forma de tablas, en las que se han indicado para cada nivel las abundancias de cada especie por cuadrícula; de este modo, hay tantas tablas como niveles, desde el nivel superficial (más reciente) hasta el nivel XI (el más antiguo) (Lozano-Francisco et al., 2003). En otra tabla se ha contabilizado el número de especies por cada nivel, observándose un gradiente en el número de especies, en la abundancia y selección de especies; en general, los niveles más antiguos son más pobres en malacofauna, mientras que en los ni- veles XII y XIII no se recuperaron moluscos (Lozano-Francisco et al., 2003).

Para la sistemática de invertebrados seguimos las clasificaciones de Vaught (1989) a nivel mundial y Sabelli et al. (1990) para el Mediterráneo; para la identificación a nivel de especie se consultó una variada bibliografía: los textos básicos consultados han sido D'Angelo y Gargiullo (1987), Consolado Macedo et al. (1998) y Poppe y Goto $(1991,1993)$ para la fauna marina; Azpeitia (1929) para las Melanopsis; Hidalgo (1874 y 1884), Gasull (1975) para los gasterópodos continentales y Serrano et al. (1995) para el estudio previo de los moluscos en la Cueva de Nerja.

\section{RESULTADOS}

A nivel taxonómico hemos identificado un total de 48 especies de invertebrados (Tabla 2) procedentes de los niveles XI a I de la Sala del Vestíbulo de la Cueva de Nerja y relacionadas, en su inmensa mayoría, con las distintas ocupaciones antrópicas representadas en estos niveles.

Del conjunto de especies, 45 son moluscos, dos son crustáceos y una es un equinodermo, sobre un total de 5.348 individuos distribuidos de forma desigual en las distintas unidades estratigráficas (Tablas 3 y 5). Un estudio detallado de la distribución de las especies de moluscos por niveles y cuadrículas en el corte del Vestíbulo puede consultarse en Lozano-Francisco et al. (2003).

El mayor número de ejemplares corresponde al nivel III (NMI: 1.109) y el mayor número de especies al nivel VIII (32).

Asimismo, en la Tabla 3 se aporta el porcentaje de especies y ejemplares recolectados por cada unidad.

Tabla 2. Sistemática de invertebrados identificados en la Sala del Vestíbulo/1962-1963.

Nota.- Iberus alonensis (Férussac, 1821) ha sido propuesto como subespecie o ecotipo de Iberus gualterianus (Linné, 1758) por Cobos (1979) y López-Alcántara et al. (1985) en base a la genitalia y a la existencia de formas intermedias en tres sierras andaluzas; según García San Nicolas (1957) existe hibridación entre ambas especies. Nuestra postura es de considerarlas especies distintas pues en las áreas geográficas estudiadas (litoral malacitano) sólo aparece I. alonensis, claramente diferenciable morfológicamente de I. gualterianus por la ausencia de carena, patrón de coloración y morfología, especie, esta última, propia de regiones montañosas. Por otro lado, sería necesario conocer si la hibridación entre ambas especies da lugar a híbridos fértiles. Tampoco se han comparado las rádulas. La similitud de los aparatos reproductores indicaría la similaridad filogenética entre ambas especies. Respecto de Iberus marmoratus (Férussac, 1821) se diferencia morfológicamente de I. alonensis por su menor talla y diferente patrón de coloración. No ha aparecido Iberus gualterianus en ninguno de los yacimientos arqueológicos del litoral malacitano (Sur de España).

Systematic of invertebrates indentified in Sala del Vestíbulo/1962-1963.

Comment: Iberus alonensis (Férussac, 1821) has been proposed as a subspecies or ecotype of Iberus gualterianus (Linné, 1758) by Cobos (1979) and López-Alcántara et al. (1985), must a similar genitalia and intermediate morphologies between three Andalucian Mountains; fide García San Nicolas (1957) I. alonensis and I. gualterianus could hibridate. Our position about them is two different species because there is one species only in the distribution studied (Málaga litoral): I. alonensis, with a different morphology of I. gualterianus, p. e. the ausence of carina, a different coloration pattern and morphology, this species live only in rocks in Spanish height mountains. Thus, it is necessary to know the hibridation between both species with fertil hibrids. The radules neither have been compared. The genitalia proposes an important philogenetic similarity between both species. Iberus marmoratus (Férussac, 1821) differs of I. alonensis for a lesser size and coloration pattern. The species Iberus gualterianus has not found in archaeologic outcrops of Málaga litoral (Southern Spain). 


\begin{tabular}{|c|c|c|c|c|c|}
\hline Unidad & Estratos & NMI & $\%$ NMI & $\mathbf{N}^{\circ}$ Especies & $\%$ Especies \\
\hline Unidad 5 & I-II-III & 2.010 & 38,16 & 33 & 26,00 \\
\hline Unidad 4 & IV & 1.065 & 20,22 & 28 & 22,05 \\
\hline Unidad 3 & V-VI-VII & 1.442 & 27,38 & 29 & 22,83 \\
\hline Unidad 2 & VIII-IX-X & 723 & 13,72 & 35 & 27,56 \\
\hline Unidad 1 & XI-XII-XIII & 23 & 0,44 & 2 & 1,58 \\
\hline
\end{tabular}

Tabla 3. Número mínimo de individuos y número de especies en valores absolutos y en porcentajes. En esta tabla no está incluido el nivel superficial.

Lower number of specimens, number of the species and percentage by levels. It is not included the superficial level.

Por otra parte, hemos agrupado las distintas especies identificadas según su hábitat, dato que nos aporta información sobre el lugar y forma de captura. Así, se han considerado 5 ecosistemas diferentes:

a) Litoral rocoso (LR), que agrupa tanto a las especies que viven en el nivel intertidal como infralitoral rocoso, ya que implica la captura en la zona litoral rocosa sobre rocas que pueden estar emergidas o permanentemente sumergidas, a poca profundidad, realizándose las capturas por métodos de marisqueo.

b) Infralitoral arenoso-fangoso (IAF), fauna siempre sumergida, pero a poca profundidad, en arenas o fangos (enterrados siempre en el caso de los bivalvos). Para su recolección son necesarios instrumentos $a d$ hoc, un "rastro" o similar.

c) Fauna de aguas abiertas que generalmente viven nadando libremente (LN) y son capturados en aguas relativamente profundas. En la actualidad se capturan con redes de arrastre ya que habitan desde pocos metros hasta $248 \mathrm{~m}$ de profundidad, aunque generalmente viven entre 25 y $30 \mathrm{~m}$.

En la colección analizada el número de especies de libres nadadores es bajo, lo que podría indicar que su captura no era selectiva, es decir, que no se utilizaran redes especialmente destinadas a este fin sino que los ejemplares se obtenían de forma esporádica, a partir de embarcaciones dedicadas a la pesca de otras especies; práctica

\begin{tabular}{|l|c|c|c|c|c|c|c|c|c|c|}
\hline & \multicolumn{2}{|c|}{ Unidad 1 } & \multicolumn{2}{c|}{ Unidad 2 } & \multicolumn{2}{c|}{ Unidad 3 } & \multicolumn{2}{c|}{ Unidad 4 } & \multicolumn{2}{c|}{ Unidad 5 } \\
\hline Hábitat & N.Esp. & $\%$ & N.Esp. & $\%$ & N.Esp. & $\%$ & N.Esp. & $\%$ & N.Esp. & $\%$ \\
\hline LR & 1 & $48 \%$ & 20 & $45 \%$ & 19 & $60 \%$ & 17 & $60 \%$ & 20 & $49 \%$ \\
\hline IAF & 0 & $0 \%$ & 9 & $18 \%$ & 5 & $17 \%$ & 5 & $14 \%$ & 10 & $15 \%$ \\
\hline LN & 0 & $0 \%$ & 3 & $8 \%$ & 1 & $3 \%$ & 1 & $4 \%$ & 3 & $8 \%$ \\
\hline F & 0 & $0 \%$ & 1 & $3 \%$ & 0 & $0 \%$ & 1 & $4 \%$ & 1 & $3 \%$ \\
\hline PT & 1 & $48 \%$ & 7 & $24 \%$ & 5 & $20 \%$ & 4 & $18 \%$ & 6 & $25 \%$ \\
\hline
\end{tabular}

Tabla 4. Número de especies y porcentaje por unidad y hábitat. LR: litoral rocoso; IAF: infralitoral arenosofangoso; LN: libres nadadores, F: fluvial; PT: pulmonado terrestre. N. Esp.: Número de especies. Number of species and percentage by level and environment. LR: rock-litoral; IAF: infralitoral sand-mud; $L N$ : free-swimming epifaunal bivalves; F: shallow water snails; PT: land snails. N. Esp.: number of species. evidenciada de forma indirecta por los estudios sobre la evolución de las tallas de los principales taxones ícticos realizados por Roselló et al. (1995).

d) Especies fluviales (F), de aguas bien oxigenadas, ríos, manantiales o arroyos, siempre con aguas dulces en movimiento.

e) Pulmonados terrestres (PT).

Cuando se analiza la distribución diacrónica de las distintas especies que representan estos distintos ecosistemas, encontramos diferencias porcentuales a lo largo de la secuencia (Tabla 4, Fig. 2).

\section{DISCUSIÓN}

Se ha identificado un NMI de 5.348 individuos distribuidos en 48 especies de invertebrados (45 de moluscos, una de equinodermo y dos de crustáceos) en la colección arqueológica procedente de los estratos I a XI ( $c a .21 .000$ - ca. 6.000 B.P.) (Pleistoceno Superior-Holoceno) de las excavaciones de 1962-1963 en la Sala del Vestíbulo de la Cueva de Nerja.

El contingente mayor de especímenes procede del nivel III (NMI: 1.108), mientras que el mayor número de especies diferentes corresponde al nivel VIII (32).

Cuando se agrupan los niveles en unidades estratigráficas (Tabla 1), observamos que el mayor número de ejemplares procede de la Unidad 5 (2.009), mientras que la mayor diversidad específica corresponde a la Unidad 2 (35 especies).

En el conjunto de la malacofauna predominan las especies de los géneros Patella y Mytilus para la fauna marina e Iberus para la terrestre.

El conjunto malacológico analizado procede de tres ambientes fundamentales: litoral rocoso, infralitoral arenoso y/o fangoso algo más profundo y por último continental (Tabla 4).

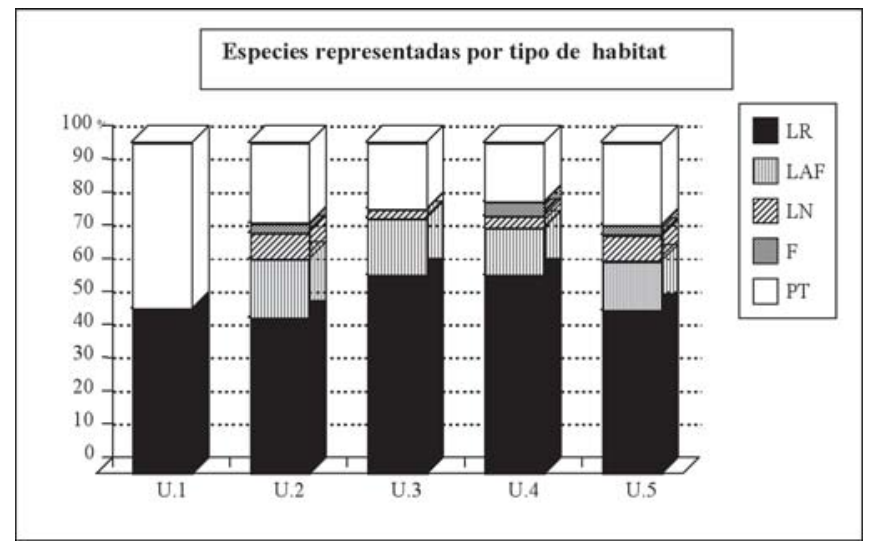

Figura 2. Porcentaje de especies representadas por tipo de hábitat (Lozano-Francisco et al., 2003).

Percentage of species and kind of environment (Lozano-Francisco et al., 2003). 


\begin{tabular}{|c|c|c|c|c|c|c|c|c|c|c|c|c|}
\hline \multirow{2}{*}{$\begin{array}{c}\text { Unidades Estratigráficas } \\
\text { Especies/Niveles }\end{array}$} & \multirow{2}{*}{$\frac{\text { Ns }}{\text { Ns }}$} & \multicolumn{3}{|c|}{ Unidad 5} & \multirow{2}{*}{$\frac{\mathrm{U.4}}{\mathrm{NIV}}$} & \multicolumn{3}{|c|}{ Unidad 3} & \multicolumn{3}{|c|}{ Unidad 2} & \multirow{2}{*}{$\begin{array}{c}\text { U.1 } \\
\text { NXI }\end{array}$} \\
\hline & & NI & NII & NIII & & NV & NVI & NVII & NVIII & NIX & $\mathbf{N X}$ & \\
\hline Antalis inaequicostatum & & & & & 2 & & & & 5 & & & \\
\hline Antalis vulgare & & & & & & & & & & 1 & & \\
\hline Glycymeris sp. & & 2 & & 2 & 5 & & & & & & & \\
\hline Mytilus edulis & 15 & 124 & 394 & 534 & 556 & 353 & 268 & 29 & 108 & 33 & 3 & 2 \\
\hline Modiolus modiolus & & & & & & & & & 4 & 1 & & \\
\hline Ostrea edule & & & 3 & 1 & 2 & & 3 & & 3 & & 1 & \\
\hline Pecten maximus & 2 & 2 & 9 & 14 & 22 & 13 & 19 & 1 & 10 & 12 & & \\
\hline Pecten jacobaeus & & & & 1 & & & & & 1 & & & \\
\hline Anomia ephippium & & & & 1 & & & & & & & & \\
\hline Rudicardium tuberculatum & & & 1 & & & 2 & 2 & & 5 & 1 & & \\
\hline Cerastoderma edule & & 1 & 6 & 8 & 9 & 7 & 22 & 2 & 44 & 9 & & \\
\hline Mactra stultorum & & & & & & & 1 & & & & & \\
\hline Ensis ensis & & & 2 & & 1 & & & & & & & \\
\hline Irus irus & & 1 & & & 1 & & & & & & & \\
\hline Tapes decussatus & & & 1 & & & 3 & 3 & & 11 & 7 & & \\
\hline Venus verrucosa & & & & & & & & & 1 & & & \\
\hline Callista chione & & 8 & 1 & & & & 1 & & & 1 & & \\
\hline Patella caerulea & 33 & 10 & 95 & 244 & 118 & 49 & 145 & 18 & 89 & 22 & 1 & \\
\hline Patella rustica & 6 & 4 & 40 & 59 & 58 & 12 & 80 & 4 & 9 & & & \\
\hline Patella nigra & & & 2 & 5 & 15 & & 13 & & 11 & 3 & & \\
\hline Patella ulyssiponensis & 5 & 16 & 37 & 93 & 66 & 18 & 45 & 2 & 25 & 2 & & \\
\hline Patella ferruginea & & & 3 & 2 & 3 & & 1 & & 1 & 1 & 1 & \\
\hline Patella intermedia & 1 & 15 & 2 & 21 & 25 & 21 & 20 & 15 & 31 & 10 & & \\
\hline Gibbula richardi & 1 & & 16 & 13 & 11 & 12 & 28 & 7 & 58 & 13 & & \\
\hline Gibbula sp. & 1 & & & & & 2 & & & 1 & & & \\
\hline Monodonta turbinata & 3 & 2 & 4 & 7 & 6 & & & 1 & 3 & 2 & & \\
\hline \multicolumn{13}{|l|}{ Littorina $\mathrm{sp}}$. \\
\hline Jujubinus exasperatus & & & & & & 1 & & & 1 & & & \\
\hline Melanopsis laevigata & 1 & 1 & & & 2 & & & & 2 & & & \\
\hline Turritella monterosatoi & & & & & & & 2 & & & & & \\
\hline Charonia lampas lampas & & & 1 & & & & 1 & & 1 & & & \\
\hline Cymatium corrugatum & & 1 & & & & & & & & & & \\
\hline Cabestana cutacea & & & & 1 & & & & & & & & \\
\hline Stramonita haemastoma & 14 & 8 & 14 & 3 & 2 & 3 & & & 1 & & & \\
\hline Nucella lapillus & & 8 & 22 & 40 & 67 & 22 & 36 & 2 & 2 & 3 & & \\
\hline Columbella rustica & & & 2 & 1 & 4 & & & & & & & \\
\hline Cyclope donovania & & & 1 & & & & & & 1 & & & \\
\hline Nassarius corniculus & & & & & & & & 1 & & & & \\
\hline Conus mediterraneus & & & & 2 & 2 & & & & & & & \\
\hline Siphonaria pectinata & 5 & 3 & 9 & 2 & 4 & & 2 & & 2 & & & \\
\hline Rumina decollata & & & & & & & & & & & 1 & \\
\hline Sphincterochila hispanica & & & & 2 & 2 & & & & 1 & 2 & & \\
\hline Otala lactea & & & & 2 & 1 & 1 & & & 1 & 2 & & \\
\hline Iberus alonensis & & 7 & 22 & 44 & 74 & 34 & 101 & 7 & 59 & 80 & 6 & 21 \\
\hline Iberus marmoratus & & 1 & 1 & 6 & 1 & 3 & 2 & & 4 & 4 & & \\
\hline Balanus sp. & & & & & 4 & 1 & & & 5 & & & \\
\hline Brachiura sp. & & & & & 2 & 1 & & & & & & \\
\hline Paracentrotus lividus & & & & & & & & & 1 & & & \\
\hline Total : 5.348 & 87 & 214 & 688 & 1108 & 1065 & 558 & 795 & 89 & 481 & 209 & 13 & 23 \\
\hline
\end{tabular}

Tabla 5. Tabla sintética del NMI por especie para niveles y unidades estratigraficas en la Sala del Vestíbulo (Lozano-Francisco et al., 2003). Ns (Nivel Superficial) no se ha tenido en cuenta por posible contaminación de la muestra.

Table of NMI/ species for levels and Chronocultural sequence of Sala del Vestibulo (Lozano-Francisco et al., 2003). Ns (Superficial level) not included in this paper because it could be contamination. 

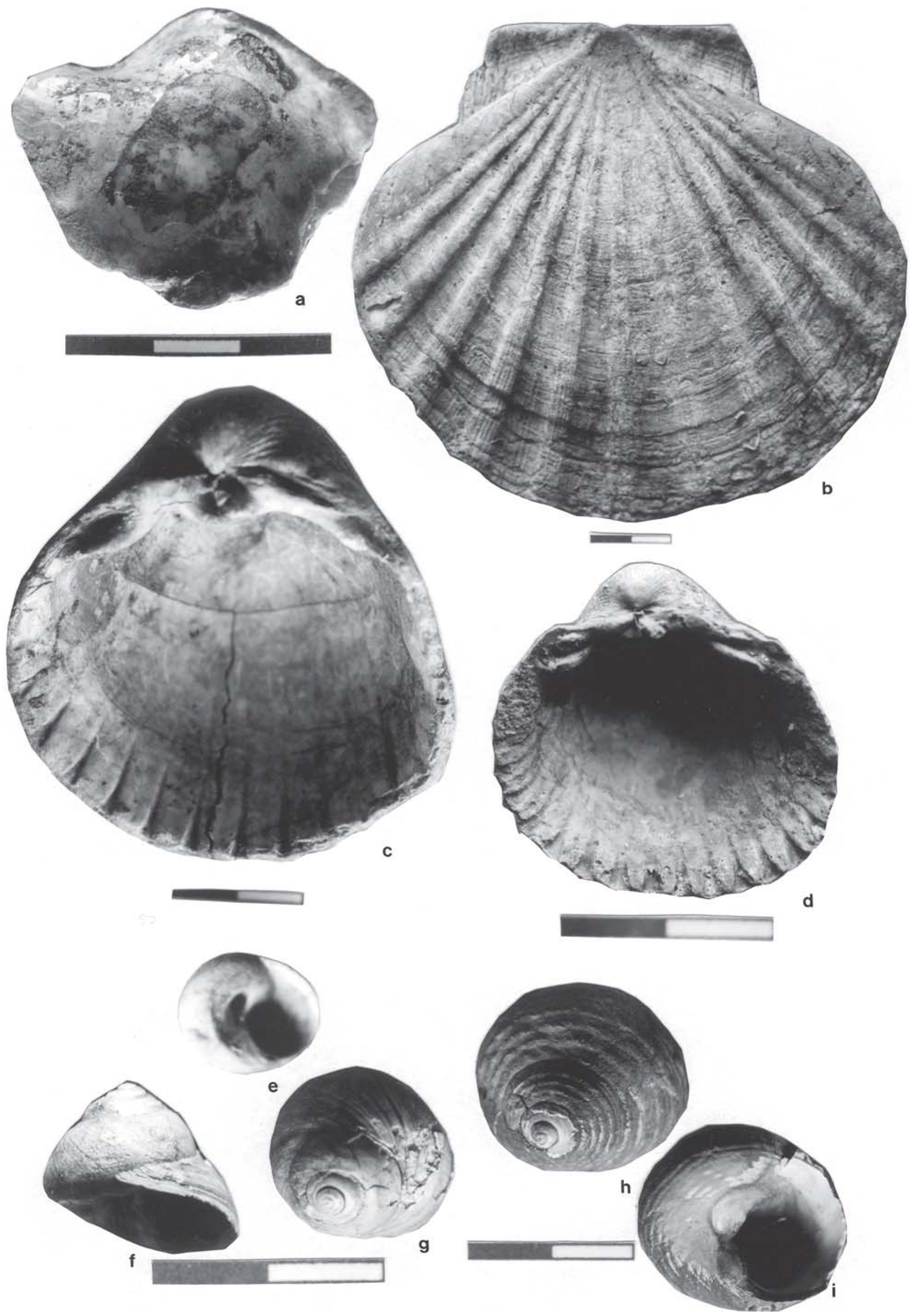
Respecto al litoral rocoso, podemos encontrar representantes del mesolitoral como patéllidos, tróquidos y el divasibranquio Siphonaria pectinata. En este medio rocoso también se incluye una zonación infralitoral poco profunda, donde se desarrollan poblaciones de patéllidos (Patella nigra y Patella ferruginea) y el murícido Stramonita haemastoma; también se incluye Columbella rustica y Conus mediterraneus.

El infralitoral arenoso y/o fangoso algo más profundo está representado por especies tales como Tapes decussatus y Cerastoderma edule y en un menor número, pero no por eso menos importante, entre otros por Jujubinus exasperatus, Nassarius corniculus, Cyllene donovania, Mactra stultorum y Callista chione.

El tercer ecosistema corresponde al medio continental, representado tanto por pulmonados, fundamentalmente por los helícidos (Iberus marmoratus e Iberus alonensis), como por gasterópodos prosobranquios fluviales del género Melanopsis. Esta presencia indicaría de un río o aguas bien oxigenadas en las proximidades de la Cueva.

Cuando se agrupan las distintas especies identificadas según su hábitat se observa que, en todos los casos, el mayor porcentaje (siempre en torno al $48 \%$ ) pertenece a fauna de litoral rocoso (Tabla 4; Fig. 2).

De las especies de litoral rocoso, es importante destacar a Mytilus edulis, presente en todos los niveles. Es una especie que vive fijada mediante un biso a sustratos duros desde la zona intertidal a la infralitoral. Su adquisición se realiza mediante métodos de marisqueo y en todos los estratos se observa que no existe selección alguna en su recolección, encontrándose desde ejemplares juveniles de pequeña talla a ejemplares de gran talla (diámetro umbo-paleal mínimo medido: $54 \mathrm{~mm}$; diámetro umbopaleal máximo medido: $105 \mathrm{~mm}$ ). Resulta también abundante el número de ejemplares de pulmonados terrestres en todos los niveles; en muchos casos se obtendrían básicamente por recolección. Respecto a las especies marinas que no se pueden obtener por el método de marisqueo, sólo las hallamos entre las unidades 2 a 5: la mayor frecuencia la encontramos en las unidades 2 y 3 (ca. 20.000 a $c a$. 11.480 B.P.), mientras que en las unidades 4 y 5 ( $c a$. 11.000 a ca. 6.000 B.P.) su presencia disminuye.

Por otro lado, es en las unidades 2 y 3 donde podemos encontrar especies tales como Jujubinus exasperatus, que se encuentra en ecosistemas de praderas de Posidonia oceanica (fanerógamas marinas), Ciclope donovania, que vive formando colonias sobre fondos de arenas finas en aguas superficiales, y Nassarius corniculus, que vive también en aguas someras sobre fondos de arenas en aguas calmadas y poco salinas.

En la Unidad 3 (ca. 12.480-11.480 B.P.) hallamos asociaciones de especies eurihalinas, es decir, que pueden soportar cambios de salinidad (Nassarius corniculus, Tapes decussatus y Cerastoderma edule), mientras que en la Unidad dos la presencia de Cyclope donovania nos indica aguas calmadas y poco profundas.

Si se realizan estudios comparativos con otras colecciones de moluscos recuperadas en las salas más exteriores (Vestíbulo, Mina y Torca) de la Cueva de Nerja (Jordá Pardo, 1981, 1982, 1983, 1984-1985, 1986; Serrano et al., 1995, 1996, 1997, 1998; Lozano-Francisco y Vera-Peláez, 1995; Lozano-Francisco et al., 2002, 2003; Vera-Peláez et al., 2003), los resultados obtenidos en nuestros análisis ratifican y profundizan en los resultados avanzados por los citados estudios.

Así, durante el Paleolítico Superior la línea de costa, con un nivel eustático relativamente bajo, estaría retirada mar adentro de la posición actual, dejando una parte de la plataforma continental emergida (Jordá Pardo, 1986; Serrano et al., 1995). En esta situación, el ambiente costero sería fundamentalmente de playa, lo que propiciaría el marisqueo de fauna infralitoral arenosa-fangosa; datos que coinciden con el registro malacológico aportado por las unidades 2 y 3 de nuestro estudio, correspondientes al Paleolítico Superior (Würm III-IV a Tardiglacial).

Estos datos coinciden por otra parte con la información disponible sobre la dinámica glacioeustática de la franja costera donde se inserta la Cueva de Nerja. Así, los estudios sobre fondos marinos en la plataforma continental de la costa de Málaga (Hernández Medina et al., 1996) ponen de manifiesto la existencia de terrazas erosivas sumergidas a diferentes isobatas $(-120,-90,-80,-73,-60$, $-45,-33$ y $-20 \mathrm{~m}$ ), de estas profundidades se podría ubicar la línea de costa a unos -120 m en torno a 20.000 B.P., mientras que la de -90 m quizás corresponda a un episodio regresivo ubicado en el Tardiglacial ( $c a .14 .000$ B.P.), mientras en ca. 12.480 B.P. la costa rondaría los $-73 \mathrm{~m}$ (correlaciones apuntadas por Aura et al., 2002 b). Según estos autores la consecuencia de estas bajadas del nivel del mar en el margen continental adyacente a la Cueva de

Figura 3. a: Anomia ephippium (Linné, 1758) en vista ventral, la escala mide $3 \mathrm{~cm}$. b: Pecten maximus (Linné, 1758), valva inferior, la escala mide $2 \mathrm{~cm}$. c: Rudicardium tuberculatum (Linné, 1758), ejemplar quemado por el fuego, vista ventral de la valva derecha, la escala mide $2 \mathrm{~cm}$. d: Cerastoderma edule (Linné, 1758), vista ventral de la valva izquierda, la escala mide $2 \mathrm{~cm}$. e, f, g: Gibbula richardi (Payraudeau, 1826) en vistas ventral, lateral y dorsal, la escala mide $2 \mathrm{~cm}$. h, i: Monodonta sp. en vistas dorsal y ventral, ejemplar quemado por el fuego.

$\boldsymbol{a}$ : Anomia ephippium (Linné, 1758), ventral view, the scale is $3 \mathrm{~cm}$. $\boldsymbol{b}$ : Pecten maximus (Linné, 1758), lower valve, the scale is $2 \mathrm{~cm}$. c: Rudicardium tuberculatum (Linné, 1758), burnt by fire, ventral view of right valve. d: Cerastoderma edule (Linné, 1758), ventral view of the left valve. $\boldsymbol{e}, \boldsymbol{f}, \boldsymbol{g}$ : Gibbula richardi (Payraudeau, 1826), ventral, lateral and dorsal view, the scale is $2 \mathrm{~cm}$. $\boldsymbol{h}$, $\boldsymbol{i}$ : Monodonta sp., dorsal and ventral view, burnt by fire, the scale is $2 \mathrm{~cm}$. 

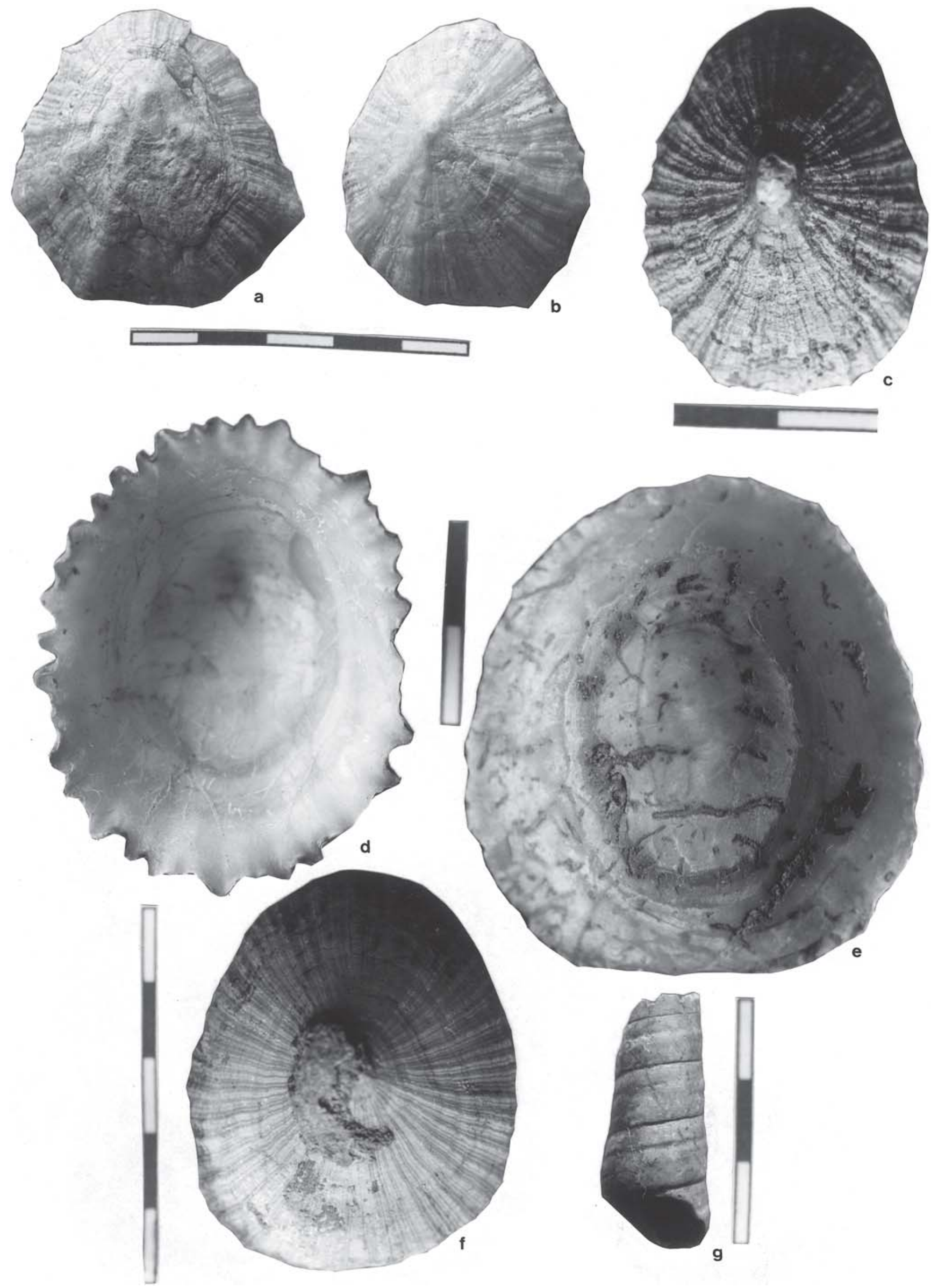
Nerja se tradujo en la emersión de una franja costera de varios kilómetros de anchura, con un mayor desarrollo de playas arenosas donde marisquear las especies de hábitat infralitoral arenoso-fangoso.

La especie Modiolus modiolus (Linne, 1758), que aparece citada por primera vez en la Cueva de Nerja en Lozano-Francisco et al. (2003) y Vera-Peláez et al. (2003), aparece asociada a fauna de mares fríos, siendo en la actualidad una especie circumboreal; es por lo tanto típicamente würmiana. Esta especie se ha encontrado en la Unidad 2 (ca. 20.000-17.000 B.P.).

Respecto a la repartición geográfica y temporal, la malacofauna estudiada es la misma que habita hoy en la provincia, a excepción de Nucella lapillus (Linne, 1758) y Modiolus modiolus, actualmente atlánticas. Así, Nucella lapillus la podemos encontrar en el Atlántico cercano, en las costas mediterráneas pertenecientes a la provincia de Cádiz, mientras que Modiolus modiolus, como ya se ha comentado, es una especie circumboreal.

En lo relativo a las posibles técnicas de obtención utilizadas, éstas dependerían en gran medida del hábitat de cada especie. Así, en la zona litoral rocosa, sobre rocas que pueden estar emergidas o permanentemente sumergidas a poca profundidad, la captura se realizaría mediante técnicas de marisqueo.

En el infralitoral arenoso-fangoso la fauna siempre está sumergida, pero a poca profundidad y sobre arenas o fangos (enterrados siempre en el caso de los bivalvos), y para su recolección es necesario algún instrumento ad hoc, generalmente un "rastro". Por último, el análisis tafonómico de las valvas de Pecten maximus (Linne, 1758) y Pecten jacobaeus (Linne, 1758), sin desgaste, abrasión o bioerosión post mortem, nos inducen a pensar que fueron capturadas vivas, mediante artes de pesca y asociadas a capturas de ictiofauna.

\section{CONCLUSIONES}

La colección analizada en este trabajo permite ampliar el repertorio taxonómico documentado hasta el momento en la Cueva de Nerja con nuevas especies (p. ej. Aequipecten inaequistatum, Modiolus modiolus, Jujubinus exasperatus, etc), confirma lo avanzado por estudios previos en cuanto que este emplazamiento constituye, tanto a nivel cuantitativo como cualitativo (NMI, biodiversidad y amplitud cronológica que ilustra), el yacimiento arqueológico más rico conocido para el estudio del aprovechamiento de recursos marinos durante el Pleistoceno Superior reciente-Holoceno antiguo en el ámbito mediterráneo peninsular. En este sentido, los estudios sobre aprovechamiento de recursos marinos que venimos realizando sobre otros yacimientos arqueológicos de la costa de Málaga (Hoyo de la Mina y Complejo del Humo, etc) permitirán aquilatar la singularidad o generalización del modelo de explotación de recursos costeros identificado en Cueva de Nerja y calibrar la incidencia y evolución del aprovechamiento en la dieta humana durante el Pleistoceno Superior y Holoceno antiguo.

Asimismo, la nueva colección permite realizar una aproximación de carácter cuantitativo a las modificaciones diacrónicas en las capturas y aprovechamientos de las distintas especies y aporta nuevos datos sobre el uso simbólico de los recursos malacológicos.

\section{AGRADECIMIENTOS}

Este estudio queda enmarcado en un proyecto de investigación auspiciado y patrocinado por la Fundación Cueva de Nerja (Revisión, estudio y contextualización cronoestratigráfica de los restos arqueológicos procedentes de las antiguas excavaciones del Patronato de la Cueva de Nerja) y que cuenta con la autorización de la Consejería de Cultura de la Junta de Andalucía y del Ministerio de Educación y Cultura.

Queremos expresar asimismo nuestro agradecimiento a la Fundación Cueva de Nerja y Museo Municipal Paleontológico de Estepona por facilitar el uso de sus dependencias para la

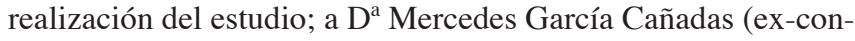
servadora del Museo Provincial de Málaga), sin cuyo celo profesional no se hubiera localizado el material que presentamos en este trabajo; a D. José Luis Barbero de Miguel, por d<ejarnos consultar su archivo personal sobre la excavación (planimetrías, anotaciones al diario de campo, fotografías, etc.) y a D. Pablo Solo de Zaldívar, quien nos suministró diversa documentación relativa a la excavación; colaboraciones y apoyos sin cuyo concurso no hubiera sido factible este estudio.

\section{BIBLIOGRAFÍA}

Aura Tortosa, J.E., Jordá Pardo, J.F., González-Tablas, J., Bécares Pérez, J. y Sanchidrián Torti, J.L. 1998. Secuencia arqueológica de la Cueva de Nerja: la Sala del Vestí-

Figura 4. a, b: Patella caerulea Linné, 1758 en vista dorsal, la escala mide $5 \mathrm{~cm}$. c: Patella ulyssiponensis Gmelin, 1791 en vista dorsal, la escala mide $2 \mathrm{~cm}$. d: Patella ferruginea Gmelin, 1791 en vista ventral, la escala mide $2 \mathrm{~cm}$. e: Patella intermedia Knapp in Murray, 1857 en vista ventral, la escala mide $2 \mathrm{~cm}$. f: Patella nigra Da Costa, 1771 en vista dorsal, la escala mide $5 \mathrm{~cm}$. g: Turritella $\mathrm{sp}$. en vista lateral, ejemplar erosionado, la escala mide $3 \mathrm{~cm}$.

$\boldsymbol{a}, \boldsymbol{b}$ : Patella caerulea Linné, 1758, dorsal view, the scale is $5 \mathrm{~cm}$. $\mathrm{c}$ : Patella ulyssiponensis Gmelin, 1791, dorsal view, the scale is $2 \mathrm{~cm}$.d: Patella ferruginea Gmelin, 1791, ventral view, the scale is $2 \mathrm{~cm}$. $\boldsymbol{e}$ : Patella intermedia Knapp in Murray, 1857, ventral view, the scale is $2 \mathrm{~cm}$. $\mathrm{f}$ : Patella nigra Da Costa, 1771, dorsal view, the scale is $5 \mathrm{~cm}$. $\mathrm{g}$ : Turritella sp. eroded, lateral view, the scale is $3 \mathrm{~cm}$. 

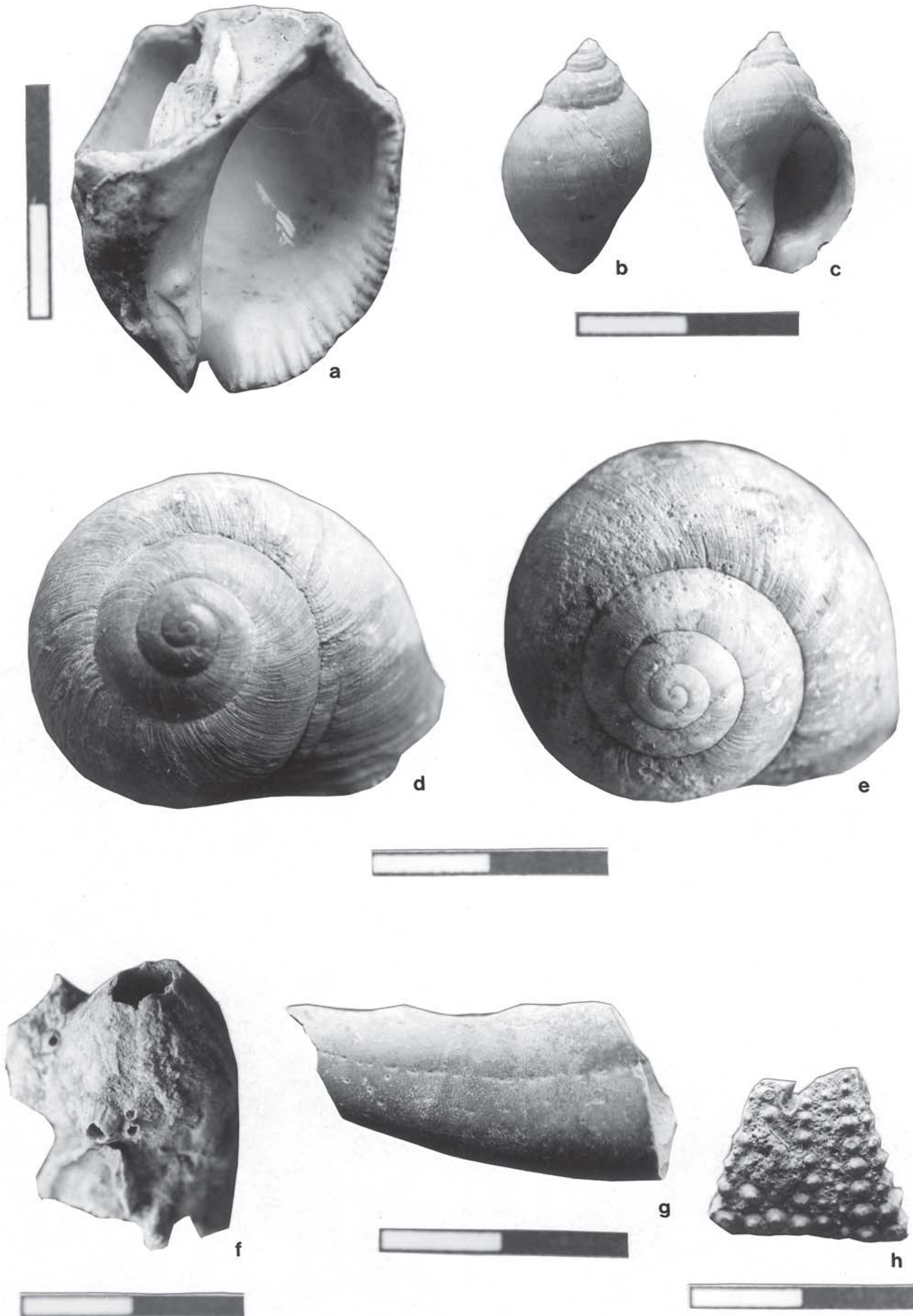
bulo. In: Las culturas del Pleistoceno superior en Andalucía (Eds. J.L. Sanchidrián y M.D. Simón). Patronato de la Cueva de Nerja, Nerja (Málaga), 217-236.

Aura Tortosa, J.E., Jordá Pardo, J.F., Pérez Ripoll, M. y Rodrigo García, M.J. 2002a. Sobre dunas, playas y calas. Los pescadores prehistóricos de la Cueva de Nerja (Málaga) y su expresión arqueológica en el tránsito Pleistoceno-Holoceno. Archivo de Prehistoria Levantina, 35, 9-39.

Aura Tortosa, J.E., Jordá Pardo, J.F., Pérez Ripoll, M., Rodrigo García, M.J., Badal García, E. and Guillem Calatayud, P. 2002b. The far south: the PleistoceneHolocene transition in Nerja Cave (Andalucía, Spain). Quaternary International, 93-94, 19-30.

Azpeitia, F. 1929. Monografía de las Melanopsis vivientes y fósiles de España. Memorias del Instituto Geológico y Minero de España, 1-403.

Cobos, A. 1979. Sobre algunos Iberus Montfort de la provincia de Almería (Gastrop. Pulm.). Boletín de la Sociedad de Historia Natural de Baleares, 23, 35-46.

Consolado Macedo, M. C., Consolado Macedo, M. e Borges, J. P. 1998. Conchas marinhas de Portugal. Ed. Verbo, Lisboa, 516 pp.

Cortés, M., Barbero, J.L., Fernández, E., Lozano-Francisco, M.C., Morales, A., Pérez, A., Riquelme, J.A., Roselló, E., Sanchidrián, J.L., Simón, M.D., Turbón, D. y Vera-Peláez, J.L. 2002. Revisión, estudio y contextualización cronoestratigráfica de los restos arqueológicos procedentes de las antiguas excavaciones del Patronato de la Cueva de Nerja. Informe técnico. Fundación Cueva de Nerja. Inédito, $381 \mathrm{pp}$.

Cotino Villa, F. y Soler Mayor, B. 1998. Ornamento sobre malacofauna. In: Las culturas del Pleistoceno superior en Andalucía (Eds. J.L. Sanchidrián y M.D. Simón). Patronato de la Cueva de Nerja, Nerja (Málaga), 301-323.

D’Angelo, G. e Gargiullo, S. 1987. Guida delle conchiglie mediterranee. Ed. Fabbri, Milán, 223 pp.

García San Nicolás, E. 1957. Estudio sobre la biología, la anatomía y la sistemática del género Iberus Montfort, 1810. Boletín de la Real Sociedad Española de Historia Natural (sección de Biología), 55, 199-390.

Gasull, L. 1975. Fauna malacológica terrestre del sudeste ibérico. Boletín de la Sociedad de Historia Natural de Baleares, 20, 1-154.
Hernández Medina, F.J., Somoza, L. and Rey, J. 1996. Late Pleistocene-Holocene High-resolution secuense analisis on the Alboran Sea continental shelf. Geological Society Special Publications, 117, 139-145.

Hidalgo, J.G. 1874. Catálogo iconográfico y descriptivo de los moluscos terrestres de España, Portugal y las Baleares. Imprenta de Segundo Martínez, $1^{\text {a }}$ entrega, Madrid, $223 \mathrm{pp}$.

Hidalgo, J.G. 1884. Catálogo iconográfico y descriptivo de los moluscos terrestres de España, Portugal y las Baleares. Establecimiento Tipográfico de E. Cuesta, $2^{\text {a }}$ entrega, Madrid, 16 pp.

Jordá Pardo, J.F. 1981. La malacofauna de la Cueva de Nerja (I). Zephyrus, 32-33, 87-99.

Jordá Pardo, J.F. 1982. La malacofauna de la Cueva de Nerja (II). Zephyrus, 34-35, 89-98.

Jordá Pardo, J.F. 1983. La secuencia malacológica de la Cueva de Nerja (Málaga). Excavaciones de 1982. Cuadernos do Laboratorios Xeóloxico de Laxe, 5, 55-71.

Jordá Pardo, J.F. 1984-1985. La malacofauna de la Cueva de Nerja (III): Evolución medioambiental y técnicas de marisqueo. Zephyrus, 37-38, 143-154.

Jordá Pardo, J.F. 1986. La fauna malacológica de la Cueva de Nerja. In: La prehistoria de la Cueva de Nerja. Trabajos sobre la Cueva de Nerja. Patronato de la Cueva de Nerja, Nerja (Málaga), 1, 145-172.

López-Alcántara, A., Rivas Carrera, P., Alonso Alonso, M.R. y Ibáñez Genis, M. 1985. Variabilidad de Iberus gualterianus (Linneo, 1758) (Pulmonata, Helicidae). Iberus, 5, 83-112.

Lozano-Francisco, M.C. y Vera-Peláez, J.L. 1995. La malacología como indicador paleoambiental y de actividad antrópica en la Cueva de Nerja (Nerja, Málaga). Comunicaciones al Congreso Internacional de Paleontología Humana, Orce (Granada), 105-106.

Lozano-Francisco, M.C., Vera-Peláez, J.L., Simón Vallejo, M.D. y Cortés Sánchez, M. 2002. Malacofauna del Pleistoceno Superior-Holoceno de la Sala del Vestíbulo, campaña 1962/63 (Cueva de Nerja, Málaga). Comunicaciones a las XVIII Jornadas de la Sociedad Española de Paleontología y II Congreso Ibérico de Paleontología. Salamanca, 66-67.

Lozano-Francisco, M.C., Vera-Peláez, J.L., Simón Vallejo, M.D. y Cortés Sánchez, M. 2003. Taxonomía, autoecología y actividad antrópica de los moluscos e invertebra-

Figura 5. a: Fragmento de Stramonita haemastoma (Linné, 1758) en vista ventral, la escala mide $2 \mathrm{~cm}$. b, c: Nucella lapillus (Linné, 1758) en vistas dorsal y ventral, la escala mide $2 \mathrm{~cm}$. d, e: Iberus alonensis (Fèrussac, 1821) en vista dorsal, d, ejemplar quemado, e, ejemplar con bioerosión producida por líquenes (Mayoral, comunicación personal), la escala mide $2 \mathrm{~cm}$. f: Balanus sp. (Crustacea, Cirripedia, Balanomorpha) en vista lateral, escala: $2 \mathrm{~cm}$. g: Pinza de cangrejo (Crustacea, Decapoda, Brachiura) en vista lateral, la escala mide $2 \mathrm{~cm} ; \mathbf{h}$ : Placas del endoesqueleto de Paracentrotus lividus (Linné, 1758) (Echinodermata, Echinozoa, Echinoida), la escala mide $2 \mathrm{~cm}$.

$\boldsymbol{a}$ : Fragment of Stramonita haemastoma (Linné, 1758), dorsal view, the scale is $2 \mathrm{~cm} . \boldsymbol{b}, \boldsymbol{c}$ : Nucella lapillus (Linné, 1758), dorsal and ventral view, the scale is $2 \mathrm{~cm}$. $\boldsymbol{d}$, $\boldsymbol{e}$ : Iberus alonensis (Fèrussac, 1821), dorsal view, $d$, specimen burnt by fire, $e$, specimen with holes done by lichens (Mayoral, personal comunication). $f$ : Balanus sp. (Crustacea, Cirripedia, Balanomorpha), lateral view, the scale is $2 \mathrm{~cm}$. g: Fragment of scrub (Crustacea, Decapoda, Brachiura), lateral view, the scale is $2 \mathrm{~cm}$. h: Fragment of skeleton of Paracentrotus lividus (Linné, 1758) (Echinodermata, Echinozoa, Echinoida), the scale is $2 \mathrm{~cm}$. 
dos marinos de la Sala del Vestíbulo (Cueva de Nerja, Málaga, Sur de España). Pliocénica, 3, 74-87.

Pellicer Catalán, M. 1997. Generalidades. In: El Neolítico y Calcolítico de la Cueva de Nerja en el contexto andaluz (Coord. M. Pellicer y P. Acosta). Trabajos sobre la Cueva de Nerja. Patronato de la Cueva de Nerja, Nerja (Málaga), 4, 15-46.

Poppe, G.T. and Goto, Y. 1991. European seashells. Vol. I (Polyplacophora, Caudofoveata, Solenogastra, Gastropoda). Ed. Verlag Christa Hemmen, Wiesbaden (Germany), $352 \mathrm{pp}$.

Poppe, G.T. and Goto, Y. 1993. European seashells. Vol. II (Scaphopoda, Bivalvia, Cephalopoda). Ed. Verlag Christa Hemmen. Wiesbaden (Germany), 221 pp.

Roselló, E., Morales, A. y Cañas, J.M. 1995. Estudio ictioarqueológico de la Cueva de Nerja (Prov. Málaga): resultados de las campañas de 1980 y 1982. In: Fauna de la Cueva de Nerja I. Salas de la Mina y de la Torca, campañas 1980-82 (Coord. M. Pellicer y A. Morales). Trabajos sobre la Cueva de Nerja. Patronato de la Cueva de Nerja, Nerja (Málaga), 5, 163-218.

Sabelli, B., Giannuzzi-Savelli, R. e Bedulli, D. 1990. Catalogo annotato dei molluschi marini del Mediterraneo, vol. 1. Librería Naturalistica Bolognese, Bolonia, 348 pp.

Sanchidrián, J.L., Simón Vallejo, M.D., Cortés, M. y Muñoz, V.E. 1996. La dinámica de los grupos predadores en la Prehistoria andaluza. In: El Paleolítico en Andalucía. Ensayo de síntesis (Editores M. Cortés, V.E. Muñoz, J.L. Sanchidrián y M.D. Simón), Córdoba, 11-93.

Serrano, F., Lozano-Francisco, M.C., Vera-Peláez, J.L. y Guerra-Merchán, A. 1995. Malacofauna en yacimientos prehistóricos de la Cueva de Nerja. In: Fauna de la Cueva de Nerja I. Salas de la Mina y de la Torca, campañas
1980-82 (Editores M. Pellicer y A. Morales). Trabajos sobre la Cueva de Nerja. Patronato de la Cueva de Nerja, Nerja (Málaga), 5, 297-373.

Serrano, F., Lozano-Francisco, M.C., Vera-Peláez, J.L. y Guerra-Merchán, A. 1996. Malacofauna en yacimientos prehistóricos de la Cueva de Nerja. I simposio de prehistoria Cueva de Nerja. Las culturas del Pleistoceno superior en Andalucía. Sección monográfica Cueva de Nerja, anexo 37, 2 pp.

Serrano, F., Guerra-Merchán, A., Lozano-Francisco, M.C. and Vera-Peláez, J.L. 1997. Multivariate analysis of remains of molluscan foods consumed by latest Pleistocene and Holocene humans in Nerja Cave (Málaga, Spain). Quaternary Research, 48, 215-227.

Serrano, F., Lozano-Francisco, M.C., Vera-Peláez, J.L. y Guerra Merchán, A. 1998. Los restos alimentarios de malacofauna de los homínidos del Pleistoceno final y Holoceno de la Cueva de Nerja (Málaga, España). In: Las culturas del Pleistoceno superior en Andalucía (Eds. J. L. Sanchidrián y M. D. Simón Vallejo). Patronato de la Cueva de Nerja, Nerja (Málaga), 359-379.

Vaught, C. 1989. A classification of the living Mollusca. American Malacologist Inc., Melbourne,189 pp.

Vera-Peláez, J.L., Lozano-Francisco, M.C., Simón Vallejo, M.D. y Cortés Sánchez, M. 2003. Relevancia del estudio de los moluscos en yacimientos prehistóricos, un caso bien documentado: la Cueva de Nerja (Málaga, Sur de España). Pliocénica, 3, 88-106.

Manuscrito recibido: 19 de diciembre, 2002 Manuscrito aceptado: 8 de junio, 2004 